\title{
Saturación de oxígeno/fracción inspirada de oxígeno como predictor de mortalidad en pacientes con exacerbación de EPOC atendidos en el Hospital Militar Central ${ }^{*}$
}

\author{
Oxygen saturation / fraction of inspired oxygen \\ as a predictor of mortality in patients with \\ exacerbation of COPD treated at the Central \\ Military Hospital ${ }^{*}$
}

\author{
Barbarita Maria Mantilla, Carlos Arturo Ramírez, \\ Sebastián Valbuena, Lilian Muñoz, Gustavo Adolfo Hincapié, \\ Alirio Rodrigo Bastidas • Bogotá, D.C. (Colombia)
}

\section{Resumen}

Introducción: la validez de la relación saturación arterial de oxígeno y fracción inspiratoria de oxígeno $\left(\mathrm{SaO}_{2} / \mathrm{FiO}_{2}\right)$, calculada por oximetría de pulso y por gases arteriales en pacientes con exacerbación de la enfermedad pulmonar obstructiva crónica (E-EPOC) a la altitud de Bogotá no son conocidos, los pacientes con EPOC pueden presentar alteraciones en el intercambio de gases que pueden empeorar con los episodios de broncoespasmo, obtener valores de la $\mathrm{SaO}_{2}$ por oximetría y $\mathrm{FiO}_{2}$ puede brindar información valiosa sobre el curso de la exacerbación.

Objetivo: determinar la validez de la relación $\mathrm{SaO}_{2} / \mathrm{FiO}_{2}$ calculada por oximetría de pulso y por gases arteriales con relación a los desenlaces de ventilación mecánica (VM) y mortalidad a siete y 30 días.

Métodos: se realizó un estudio de cohorte prospectivo con análisis de prueba diagnóstica calculando los puntajes DECAF, BAP-65, CURB-65, gases arteriales y oximetría de pulso al ingreso de pacientes con E-EPOC, se evaluó el desenlace de mortalidad a los siete y 30 días de ingreso y el requerimiento de VM durante su hospitalización, se calculó la relación $\mathrm{SaO}_{2} / \mathrm{FiO}_{2}$ utilizando la $\mathrm{SaO}_{2}$ obtenida en los gases arteriales y de manera independiente la relación $\mathrm{SaO}_{2} / \mathrm{FiO}_{2}$ con la $\mathrm{SaO}_{2}$ obtenida por oximetría de pulso, con los datos obtenidos se calculó los valores de sensibilidad (S), especificidad (E), valor predictivo positivo (VPP), valor predictivo negativo (VPN), razón de verosimilitud positiva (LR+), razón de verosimilitud negativa (LR-) y área bajo la curva de características operativas del receptor (ACOR).

Resultados: se analizaron 462 E-EPOC, el requerimiento de VM fue de $14.3 \%$ y mortalidad a 30 días de $5.71 \%$, la sensibilidad de la relación $\mathrm{SaO}_{2} / \mathrm{FiO}_{2}$ calculada por oximetría de pulso para desenlace de VM fue de 84.6\% (IC95\%:75-94.2), especificidad 42\% (IC95\%:37- 47), VPP: 19.6\% (IC95\%:14.8-24.4), VPN: 94.3\% (IC95\%:90.5-98), LR+: 1.46 (IC95\%:1.28-1.67), LR: 0.37(IC95\%:0.20-1.67), ACOR: 0.779\% (IC95\%:0.711-0.847) p<0.0001, la sensibilidad de relación $\mathrm{SaO}_{2} / \mathrm{FiO}_{2}$ por gases arteriales para VM fue de 83\% (IC95\%:73.2-92.9), especificidad 57\% (IC95\%:51.9-62.2), VPP: 24.8\% (IC95\%:18.8-30.7), VPN: 95.2\% (IC95\%:92.2-98.2), LR+: 1.94 (IC95\%:1.65-2.27), LR-: 0.30 (IC95\%:0.17-0.51), ACOR: 0.799\% (IC95\%:0.737-0.861) $\mathrm{p}<0.0001$, la sensibilidad de la relación $\mathrm{SaO}_{2} / \mathrm{FiO}_{2}$ por oximetría para desenlace de mortalidad tiene una sensibilidad del 76.8\% (IC95\%:58.8-95), especificidad de 39.2\% (IC95\%:34.4-43.9), VPP: 7.1\% (IC95\%:3.9-10.3), VPN: 96.5\% (IC95\%:93.5-99.5), LR+: 1.26 (IC95\%:1.01-1.58), LR-: 0.59 (IC95\%:0.29-1.20), ACOR: 0.689\% (IC95\%:0.568-0.810) p<0.0001, la sensibilidad de la relación $\mathrm{SaO}_{2} / \mathrm{FiO}_{2}$ por gases arteriales para mortalidad fue de 80.8\% (IC95\%:63.7-97.8), especificidad 53.2\% (IC95\%:48.3-58), VPP: 9.6\% (IC95\%:5.5-13.8), VPN: 97.8\% (IC95\%:95.7-99.9),
*Trabajo ganador del Primer Puesto del Premio "Trabajo de Investigación Clínica - Residentes", otorgado por el X Congreso SOLAMI XXVI Congreso ACMI-ACP, Cartagena 17-20 de agosto de 2017.

Doctores: Barbarita María Mantilla Cardozo, MsC, Carlos Arturo Ramírez Sierra, Sebastián Valbuena Benítez, Lilian Muñoz Marrugo, Gustavo Adolfo Hincapié Díaz: Hospital Militar Central, Universidad Militar Nueva Granada; Dr. Alirio Rodrigo Bastidas Goyes, MsC: Universidad de la Sabana, Clínica Universidad de la Sabana. Bogotá, D.C. (Colombia).

Correspondencia: Dr. Alirio Rodrigo Bastidas Goyes. Bogotá, D.C. (Colombia).

E-mail: alirio.bastidas@unisabana.edu.co, alirio.bastidas@clínicaunisabana.edu.co, Recibido: 30/VI/2017 Aceptado: 19/VIII/2017 
LR+: 1.73 (IC95\%:1.39-2.14), LR-: 0.36 (IC95\%:0.16-0.80), ACOR: 0.732\% (IC95\%:0.617$0.846) \mathrm{p}<0.0001$.

Conclusión: los valores de $\mathrm{SaO}_{2} / \mathrm{FiO}_{2}$ calculados por oximetría y por gases arteriales pueden ser útiles para predecir desenlaces de VM y mortalidad en pacientes con exacerbación de la EPOC. (Acta Med Colomb 2017; 42: 215-223).

Palabras clave: saturación arterial de oxígeno, enfermedad pulmonar obstructiva crónica, sensibilidad y especificidad, puntaje.

\section{Abstract}

Introduction: the validity of the ratio arterial oxygen saturation and inspiratory oxygen fraction $\left(\mathrm{SaO} / \mathrm{FiO}_{2}\right)$, calculated by pulse oximetry and by arterial gases in patients with exacerbation of chronic obstructive pulmonary disease (E-COPD) at the altitude of Bogotá are not known. Patients with COPD can present alterations in gas exchange that can worsen with bronchospasm episodes. Obtaining values of $\mathrm{SaO}_{2}$ by oximetry and $\mathrm{FiO}_{2}$ can provide valuable information on the course of the exacerbation.

Objective: to determine the validity of the $\mathrm{SaO}_{2} / \mathrm{iO}_{2}$ ratio calculated by pulse oximetry and by arterial gases in relation to mechanical ventilation (VM) outcomes and mortality at seven and 30 days.

Methods: a prospective cohort study with diagnostic test analysis was performed, calculating DECAF, BAP-65, CURB-65, arterial blood gas and pulse oximetry scores on admission of patients with E-COPD, and the outcome of mortality was evaluated at seven and 30 days of admission and the MV requirement during hospitalization; the $\mathrm{SaO}_{2} / \mathrm{FiO}_{2}$ ratio was calculated using the $\mathrm{SaO}_{2}$ obtained in the arterial gases and independently the $\mathrm{SaO}_{2} / \mathrm{FiO}_{2}$ ratio with the $\mathrm{SaO}_{2}$ obtained by pulse oximetry. With the data obtained, the values of sensitivity (S), specificity (E), positive predictive value (VPP), negative predictive value (NPV), positive likelihood ratio $(\mathrm{LR}+)$, negative likelihood ratio (LR-) and low area the operating characteristics curve of the receiver (ACOR) were calculated.

Results: 462 E-COPD were analyzed; the MV requirement was $14.3 \%$ and 30-day mortality of $5.71 \%$, the sensitivity of the $\mathrm{SaO}_{2} / \mathrm{FiO}_{2}$ ratio calculated by pulse oximetry for MV outcome was $84.6 \%$ (95\% CI : 75-94.2), specificity $42 \%$ (95\% CI: 37-47), PPV: $19.6 \%$ (95\% CI: 14.8-24.4), NPV: $94.3 \%$ (95\% CI: 90.5-98), LR +: 1.46 (95\% CI : 1.28-1.67), LR: 0.37 (95\% CI: 0.20-1.67), ACOR: $0.779 \%$ (95\% CI: 0.711-0.847) p <0.0001, sensitivity of $\mathrm{SaO}_{2} / \mathrm{FiO}_{2}$ ratio for arterial blood gases for MV was $83 \%$ (95\% CI: 73.2-92.9), specificity 57\% (95\% CI: 51.9-62.2), PPV: $24.8 \%$ (95\% CI: 18.8-30.7), NPV: 95.2\% (95\% CI: 92.2-98.2), LR +: 1.94 (IC95\%: 1.65-2.27), LR-: 0.30 (IC95\%: 0.17-0.51), ACOR: $0.799 \%$ (IC95\%: 0.737-0.861) p <0.0001, the sensitivity of the $\mathrm{SaO}_{2} / \mathrm{FiO}_{2}$ ratio by oximetry for outcome of mortality has a sensitivity of $76.8 \%$ (95\% CI: 58.8 95), specificity of $39.2 \%$ (95\% CI: 34.4-43.9), PPV: 7.1\% (95\% CI: 3.9-10.3), NPV: $96.5 \%$ (95\% CI : 93.5-99.5), LR +: 1.26 (IC95\%: 1.01-1.58), LR-: 0.59 (CI 95\%: 0.29-1.20), ACOR: 0.689\% (IC95\%: 0.568-0.810) $\mathrm{p}<0.0001$, the sensitivity of the $\mathrm{SaO}_{2} / \mathrm{FiO}_{2}$ ratio for arterial blood gases was 80.8\% (95\% CI: 63.7-97.8), specificity 53.2\% (95\% CI: 48.3-58), PPV: 9.6\% (95\% CI: 5.5-13.8), NPV: $97.8 \%$ (95\% CI: 95.7-99.9), LR +: 1.73 (95\% CI: 1.39-2.14), LR-: 0.36 (IC95\%: 0.16-0.80), ACOR: $0.732 \%$ (IC95\%: 0.617-0.846) p <0.0001.

Conclusion: $\mathrm{SaO}_{2} / \mathrm{FiO}_{2}$ values calculated by oximetry and arterial blood gases can be useful to predict MV outcomes and mortality in patients with exacerbation of COPD. (Acta Med Colomb 2017; 42: 215-223).

Key words: arterial oxygen saturation, chronic obstructive pulmonary disease, sensitivity and specificity, score.

\section{Introducción}

Según la WHO (World Health Organization) la enfermedad pulmonar obstructiva crónica (EPOC) es la cuarta causa de mortalidad a nivel global, con proyección a 2020 a ser la tercera (1); dependiendo de la severidad de la enfermedad, los pacientes con diagnóstico de EPOC cursan con una a cuatro exacerbaciones por año, pero se considera hay un importante subregistro de los episodios de exacerbación que depende en muchos casos del lugar de atención y del manejo que se instaure al paciente (2). A pesar de la fisiopatología de la enfermedad, no todas las exacerbaciones requieren hospitalización, muchas son manejadas de forma ambulatoria con ajuste de medicamentos o inicio de los mismos con buena respuesta; por otra parte, 
dichas exacerbaciones son causa frecuente de ingreso a cuidados intensivos, instauración de ventilación mecánica invasiva y no invasiva, y mortalidad (3).

El amplio y extremo espectro de manejo de la enfermedad hace necesario tener claros marcadores clínicos y paraclínicos que permitan predecir el comportamiento de la exacerbación y por ende orienten la conducta. Sin embargo, los puntajes predictores con que se cuenta en la actualidad implican la realización de estudios no siempre disponibles en todos los niveles de atención, eventualmente conllevando a dificultades en la toma de decisiones con respecto a la atención, evolución y remisión.

Los puntajes más reconocidos, estudiados, validados y utilizados en la actualidad, para predecir desenlace en exacerbación de EPOC son el DECAF, CURB 65 y BAP 65 (4-6) (3-6), los cuales de forma general implican la realización de los siguientes paraclínicos: conteo de eosinófilos, electrocardiograma, radiografía de tórax, gases arteriales, nitrógeno ureico. Y si utilizamos puntajes como APACHE 2 los requerimientos de paraclínicos son aún más exigentes (7). Dichos paraclínicos no están disponibles de forma amplia en todos los escenarios de atención de pacientes con exacerbación de EPOC, y adicionalmente su interpretación se ve limitada por la experticia del clínico que los analiza.

Es así como se requiere una variable clínica accesible, segura, económica y fácil de interpretar que permita al clínico, en escenarios de carencia de apoyo logístico y tecnología, una toma de decisiones más acertada para el paciente. El presente estudio se plantea con el objetivo de determinar si la saturación arterial de oxígeno en relación con la fracción inspirada de oxígeno $\left(\mathrm{SaO}_{2} / \mathrm{FiO}_{2}\right)$, sirve como variable independiente para predecir mortalidad y uso de ventilación mecánica en pacientes con exacerbación aguda de EPOC a una altura de $2600 \mathrm{msnm}$, como elemento económico, seguro y accesible de medición.

\section{Material y métodos}

Se realizó un estudio de cohorte prospectivo con análisis de prueba diagnóstica con toma de información de variables para el cálculo de los puntajes DECAF, BAP-65, CURB-65, gases arteriales, oximetría al ingreso y seguimiento a 30 días, en pacientes con exacerbación de EPOC (E-EPOC) del 17 de febrero de 2014 al 17 de enero de 2017 que consultarán al servicio de urgencias del Hospital Militar Central, se definió como exacerbación de EPOC el evento que genera un cambio agudo de la condición basal del paciente con EPOC caracterizado por aumento de la tos, disnea, volumen y purulencia del esputo que requiere ajuste de la medicación basal, se evaluaron desenlaces de mortalidad a los siete y 30 días de ingreso y el requerimiento de ventilación mecánica (invasiva o no invasiva). Los cálculos de los puntajes de DECAF, BAP-65 y CURB-65, se realizó según los estudios originales de validación para cada uno de ellos, se calculó la relación $\mathrm{SaO}_{2} / \mathrm{FiO}_{2}$ utilizando la $\mathrm{SaO}_{2}$ obtenida en los gases arteriales y de manera independiente la relación $\mathrm{SaO}_{2} / \mathrm{FiO}_{2}$ con la $\mathrm{SaO}_{2}$ obtenida por oximetría.

Se incluyeron pacientes mayores de 40 años, de ambos sexos, con antecedentes de EPOC diagnosticada con criterios espirométricos o diagnóstico presuntivo de la misma con diagnóstico primario de ingreso de exacerbación de la EPOC (CIE 10 J440 - J441), fumador de más de 10 paquetes/año y/o exposición a biomasa por más de 10 años, se excluyeron pacientes con antecedente de hospitalización los 30 días previos por cualquier causa, pacientes no candidatos a unidad de cuidados intensivos por criterios clínicos, comorbilidad esperada para limitar supervivencia menos de 12 meses, estado terminal, infarto agudo de miocardio diagnosticado al ingreso y pacientes con neumotórax asociado al ingreso a urgencias.

El tamaño de muestra se realizó con el programa estadístico EPIDAT (Versión 3.0), para un estudio de prueba diagnóstica considerándose una sensibilidad de $80 \%$, especificidad de $90 \%$ y precisión de $10 \%$, y un porcentaje de pérdida de $20 \%$ un mínimo de 462 eventos (exacerbaciones de EPOC), se utilizó un muestreo por conveniencia para el ingreso de los pacientes. Se incluyeron en el estudio variables sociodemográficas, antecedentes patológicos, historia de exposición a cigarrillo, signos vitales, síntomas respiratorios, grado de disnea, función renal, cuadro hemático, gases arteriales, electrocardiograma y radiografía de tórax.

En el análisis de datos se utilizó el programa SPSS versión 20 licenciado, las variables cuantitativas se resumieron dependiendo de su distribución, en promedio y desviación estándar para distribución normal y mediana y rango intercuartílico para las de distribución no normal, las variables cualitativas se resumieron en frecuencias y porcentajes, se realizó análisis bivariado con t de student y u de Mann Witney para variables comparación de promedios o medianas y chi ${ }^{2}$ para variables cualitativas, se consideró una $\mathrm{p}<0.2$ para ser incluida en el análisis multivariado, se realizó una regresión logística para los desenlaces de mortalidad y ventilación mecánica tomándose un valor p estadísticamente significativo $<0.05$ en el modelo, se realizó posteriormente el cálculo del área bajo la curva operativa del receptor (ACOR), sensibilidad (S), especificidad (E), valor predictivo positivo (VPP), valor predictivo negativo (VPN), razón de verosimilitud positiva (LR+), razón de verosimilitud negativa (LR-) para los desenlaces de muerte y ventilación mecánica con sus intervalos de confianza de $95 \%$ y valor $p$.

Este trabajo se desarrolló teniendo en cuenta la reglamentación ética vigente (Declaración de Helsinki/ Reporte Belmont/ Pautas CIOMS y Resolución 8430 de 1993), considerándose un estudio sin riesgo. Los datos obtenidos se emplearon única y exclusivamente como objeto de estudio científico y tuvieron un manejo estrictamente confidencial, el trabajo fue aprobado por el comité de ética del Hospital Militar Central sin requerirse consentimiento informado. 


\section{Resultados}

En total se incluyeron en el estudio 462 exacerbaciones de la enfermedad pulmonar obstructiva crónica que consultaron al servicio de Urgencias del Hospital Militar Central, el requerimiento de ventilación mecánica (invasiva y no invasiva) fue de $14.3 \%$ y de mortalidad a 30 días de $5.71 \%, 50 \%$ sexo masculino, el promedio de edad de los pacientes fue de 79 años y los antecedentes referidos con más frecuencia fueron hipertensión y falla cardiaca. El síntoma más frecuentemente referido fue incremento de la disnea en $97 \%$ de los pacientes y la mayoría de los pacientes tenía un estado de conciencia, medido por escala de Glasgow normal. En la Tabla 1 se describen las características generales de la población y el desenlace de ventilación mecánica.

\section{Requerimiento de ventilación mecánica}

Con relación al desenlace requerimiento de ventilación mecánica en algún momento de la estancia no se encontró diferencia significativa entre el sexo de los pacientes, edad o la presencia de los principales antecedentes patológicos (Tabla 1). Así mismo no se observó diferencia entre los síntomas que refería el paciente de acuerdo con las variables analizadas por el puntaje de Anthonisen y la necesidad de instaurar soporte ventilatorio. Los signos vitales de los pacientes con diferencia estadística para ventilación mecánica fueron la frecuencia cardiaca y la frecuencia respiratoria, no se encontró diferencia en las cifras de presión arterial. El estado ácido base sí tuvo una relación con la instauración de la ventilación mecánica siendo en promedio el pH de 7.32 en pacientes que se ventilaron, de igual manera se relacionan los niveles de $\mathrm{PaCO}_{2}$ siendo de $36.9 \mathrm{mmHg}$ en promedio para los pacientes no ventilados y de 46.9 para los ventilados $(\mathrm{p}<0.001)$ (Tabla 1).

No se obtuvieron diferencias estadísticamente significativas en la saturación de oxígeno para identificar pacientes con necesidad de soporte ventilatorio. $\mathrm{La} \mathrm{SaO}_{2}$ por gases arteriales tampoco mostró diferencia estadística, siendo de $90.7 \%$ en los pacientes no ventilados y $88.9 \%$ en los ventilados ( $\mathrm{p}=0.063$ ) en las mediciones por gases arteriales, $\mathrm{y}$ $89.9 \%$ y $89.7 \%$ ( $\mathrm{p}=0.857$ ) para los ventilados y no ventilados cuando se medía por pulso oximetría. Si bien las variables individuales no tuvieron diferencia estadísticamente significativa, las variables en relación con el aporte de oxigeno que tenía el paciente $\left(\mathrm{FiO}_{2}\right)$, sí la $\mathrm{PaO}_{2} / \mathrm{FiO}_{2}$ en los pacientes no ventilados fue en promedio de 252 y en los ventilados de $191(\mathrm{p}<0.0001)$. $\mathrm{La} \mathrm{SaO}_{2} / \mathrm{FiO}_{2}$ en gases arteriales fue de 331 en promedio para pacientes ventilados y de 258 en pacientes no ventilados con una $\mathrm{p}<0.0001$ y la $\mathrm{SaO}_{2} / \mathrm{FiO}_{2}$ medida con pulso oxímetro fue de 331 en los pacientes que no se ventilaron y de 258 en los que sí se ventilaron $(\mathrm{p}<0.0001)$ (Tabla 1).

\section{Mortalidad}

La característica demográfica de los pacientes con mortalidad a siete y 30 días no fue diferente al grupo que sobrevi- vió a los 30 días de la exacerbación. No hay diferencias de edad y sexo; las comorbilidades tuvieron un comportamiento similar, excepto la presencia de falla cardiaca que fue prevalente de forma estadísticamente significativa $(\mathrm{p}<0.0001)$ en los pacientes con mortalidad a 30 días (Tabla 2).

En los hallazgos clínicos no hubo relación entre los cambios en el esputo en cuanto a volumen y características o la presencia de disnea que se relacionaran en forma significativa con la mortalidad a siete y 30 días. La variable clínica en relación con el estado de conciencia medido con la escala de Glasgow sí tuvo significancia. Los pacientes con Glasgow de 10 o menos tuvieron mayor mortalidad con una $\mathrm{p}<0.001$. La taquicardia se relacionó con mayor frecuencia con el desenlace fatal $(\mathrm{p}<0.0001$ y 0.004$)$ (Tabla 2). El pH y los niveles de $\mathrm{PaCO}_{2}$ se relacionaron con mortalidad a 30 días. No hubo diferencia en la oximetría de pulso en relación con mortalidad, pero sí se encontró relación con la $\mathrm{SaO}_{2} / \mathrm{FiO}_{2}$ medida en gases arteriales para mortalidad a siete días $(325$ y 240 con $\mathrm{p}<0.0001)$ y a 30 días (326 y 267 con p<0.0001). $\mathrm{La} \mathrm{SaO}_{2} / \mathrm{FiO}_{2}$ calculada con el oxímetro de pulso se relacionó con la muerte a 30 días identificándose valores promedio de 323 en los pacientes que sobreviven y 277 en los que mueren $(p<0.0001)$. Dentro de los paraclínicos el único que se relacionó con la mortalidad fue el nivel de nitrógeno ureico, siendo de 21.4 vs 32.9 en los pacientes con mortalidad a siete días $(\mathrm{p}<0.0001)$ y de $21.2 \mathrm{vs} 29.7 \mathrm{mg} / \mathrm{dL}$ en los pacientes con mortalidad a 30 días $(\mathrm{p}<0.0001)$.

\section{Puntajes de severidad}

Todos los puntajes evaluados DECAF, BAP-65 y CURB65 se relacionaron con los desenlaces de ventilación mecánica y mortalidad, los resultados de la relación de los puntajes con los desenlaces de estudio se muestran en la Tabla 3.

\section{Rendimiento de la relación $\mathrm{SaO}_{2} / \mathrm{FiO}_{2}$ de gases arteriales y con pulso oximetría}

Los resultados del análisis multivariado de regresión logística para ventilación mecánica obtuvieron diferencias estadísticamente significativas para la frecuencia respiratoria $(\mathrm{p}=0.015), \mathrm{pH}(\mathrm{p}=0.007), \mathrm{PaCO}_{2}(\mathrm{p}=0.004)$ y $\mathrm{SaO}_{2} / \mathrm{FiO}_{2}$ $(\mathrm{p}<0.0001)$, y para mortalidad fueron edad $(\mathrm{p}=0.034), \mathrm{ICC}$ $(\mathrm{p}=0.004)$, Glasgow $(\mathrm{p}=0.06), \mathrm{PaCO} 2(\mathrm{p}=0.015), \mathrm{SaO}_{2} /$ $\mathrm{FiO}_{2}(\mathrm{p}=0.002)$ y BUN $(\mathrm{p}=0.031)$. La correlación entre $\mathrm{SaO}_{2}$ tomada por gases y $\mathrm{SaO}_{2}$ tomada por pulso oximetría valorada con el coeficiente de correlación de Pearson fue del 0.067 (IC95\%:-0.005-0.159) p=0.156, y la correlación para $\mathrm{SaO}_{2} / \mathrm{FiO}_{2}$ calculada por gases y $\mathrm{SaO}_{2} / \mathrm{FiO}_{2}$ calculada por pulso oximetría fue del 0.881 (IC95\%:0.839-0.918) $\mathrm{p}<0.0001$. Los valores de sensibilidad, especificidad, área bajo la curva de características operativas del receptor para $\mathrm{SaO}_{2}$ obtenida a través de gases arteriales y por pulso oximetría fueron bajos, con una ACOR que sobrepasa línea de referencia 0.5, sin embargo, el ACOR, sensibilidad y especificidad de la relación $\mathrm{SaO}_{2} / \mathrm{FiO}_{2}$ por gases arteriales 
TRABAJOS ORIGINALES • $\mathrm{SO}_{2} / \mathrm{FIO}_{2}$ como predictor de mortalidad en EPOC

Tabla 1. Características de la población con desenlace ventilación mecánica (VM)

\begin{tabular}{|c|c|c|c|c|}
\hline Variable & Población total $n=462$ & No VM n=396 & $V M n=66$ & valor $p$ \\
\hline Edad años x(ds) & $79(8.3)$ & $79(8.5)$ & $78.6(7)$ & 0.382 \\
\hline Sexo masculino $\mathrm{n}(\%)$ & $233(50.4)$ & $198(50)$ & $35(53)$ & 0.648 \\
\hline \multicolumn{5}{|l|}{ Antecedentes n(\%) } \\
\hline IAM & $69(14.9)$ & $55(13.9)$ & $14(21.2)$ & 0.122 \\
\hline $\mathrm{DM}$ & $105(22)$ & $88(22.2)$ & $17(25.8)$ & 0.526 \\
\hline ERC & $41(8.9)$ & $31(7.8)$ & $10(15.2)$ & 0.053 \\
\hline HTA & $377(81.6)$ & $321(81.1)$ & $56(84.8)$ & 0.462 \\
\hline Falla cardiaca & $135(29.2)$ & $110(27.8)$ & $25(37.9)$ & 0.095 \\
\hline \multicolumn{5}{|l|}{ Síntomas n(\%) } \\
\hline Incremento purulencia esputo & $224(48.5)$ & 193(48.7) & $31(47)$ & 0.79 \\
\hline Incremento producción esputo & $319(69)$ & $274(69.2)$ & $45(68.2)$ & 0.869 \\
\hline Incremento de la disnea & $449(97.2)$ & $385(97.2)$ & $64(97)$ & 0.9 \\
\hline \multicolumn{5}{|l|}{ Glasgow n(\%) } \\
\hline 10 & $1(0.2)$ & $1(0.3)$ & $0(0)$ & 0.159 \\
\hline 11 & $2(0.4)$ & $2(0.5)$ & $0(0)$ & \\
\hline 14 & $7(1.5)$ & $4(1)$ & $3(4.5)$ & \\
\hline 15 & $450(97.4)$ & $387(98.2)$ & $63(95.5)$ & \\
\hline \multicolumn{5}{|l|}{ Signos vitales $\mathrm{x}(\mathrm{ds})$} \\
\hline Pulso latxmin & $88.2(16)$ & $86.99(148)$ & $95.2(20.6)$ & $<0.0001$ \\
\hline FR respxmin & $20.7(3.9)$ & $20.26(3)$ & $23.21(6.8)$ & $<0.0001$ \\
\hline PAS mmHg & $135.9(21)$ & $136(20.28)$ & $135.2(25)$ & 0.764 \\
\hline PAD mmHg & $77.4(14.5)$ & $77.25(13.9)$ & $78.17(15.5)$ & 0.634 \\
\hline \multicolumn{5}{|l|}{ Paraclínicos x(ds) } \\
\hline $\mathrm{pH}$ & $7.38(0.06)$ & $7.38(0.05)$ & $7.32(0.07)$ & $<0.0001$ \\
\hline $\mathrm{PaO}_{2} \mathrm{mmHg}$ & $68.5(18.3)$ & $68.61(17.7)$ & $67.9(21.5)$ & 0.779 \\
\hline $\mathrm{PaCO}_{2} \mathrm{mmHg}$ & $38.3(9.6)$ & $36.9(7.5)$ & $46.9(14.8)$ & $<0.0001$ \\
\hline $\mathrm{HCO}_{3} \mathrm{mmol} / \mathrm{L}$ & $22.3(3.4)$ & $21.9(3.5)$ & $24.6(5.5)$ & $<0.0001$ \\
\hline $\mathrm{SaO}_{2} \%$ gases & $90.5(7.4)$ & $90.7(7.1)$ & $88.9(9)$ & 0.063 \\
\hline $\mathrm{SaO}_{2} \%$ oximetría & $89.9(6.2)$ & $89.9(6.2)$ & $89.7(5.8)$ & 0.857 \\
\hline $\mathrm{PaO}_{2} / \mathrm{FiO}_{2}$ & $243.9(73.8)$ & $252.9(70.2)$ & 191(72.8) & $<0.0001$ \\
\hline $\mathrm{SaO}_{2} / \mathrm{FiO}_{2}$ gases & $323.4(68)$ & $335(58.8)$ & $255(77.9)$ & $<0.0001$ \\
\hline $\mathrm{SaO}_{2} / \mathrm{FiO}_{2}$ oximetría & $321(65.2)$ & $331.8(56.8)$ & $258(75.5)$ & $<0.0001$ \\
\hline $\mathrm{Hb} \mathrm{mg} / \mathrm{dL}$ & $14(2.1)$ & $14.09(2.07)$ & $14.04(2.34)$ & 0.858 \\
\hline Hto $\%$ & $42.5(6.7)$ & $42.3(6.5)$ & $43.3(8.3)$ & 0.29 \\
\hline Creatinina mg/dL & $1(0.83)$ & $0.96(0.54)$ & $1.26(1.75)$ & 0.007 \\
\hline BUN mg/dL & $21.7(10.9)$ & $21.2(10.3)$ & $24.6(13.8)$ & 0.02 \\
\hline Eosinófilos $<50 \mathrm{cel} / \mathrm{mm}^{3} \mathrm{n}(\%)$ & 257(55.6) & $217(54.8)$ & $40(60)$ & 0.379 \\
\hline Consolidación n(\%) & $76(16.5)$ & $62(15.7)$ & $14(21.2)$ & 0.26 \\
\hline Fibrilación auricular n(\%) & $36(7.8)$ & $26(6.6)$ & $10(15.2)$ & 0.016 \\
\hline
\end{tabular}


B. M. Mantilla y cols.

Tabla 2. Características de la población con desenlace muerte siete y 30 días.

\begin{tabular}{|c|c|c|c|c|c|c|}
\hline & \multicolumn{3}{|c|}{ Mortalidad siete días } & \multicolumn{3}{|c|}{ Mortalidad 30 días } \\
\hline & Vivos $n=450$ & $\begin{array}{l}\text { Muertos siete } \\
n=12\end{array}$ & valor $\mathbf{p}$ & Vivos $n=435$ & Muertos $n=27$ & valor $p$ \\
\hline Edad años $\mathrm{x}(\mathrm{ds})$ & $78.8(8.3)$ & $84.8(6.5)$ & 0.013 & $78.7(8.3)$ & $82.8(6.9)$ & 0.015 \\
\hline Sexo masculino $\mathrm{n}(\%) \ldots$ & $226(50.2)$ & $7(58.3)$ & 0.579 & $219(50.3)$ & 14(51.9) & 0.879 \\
\hline \multicolumn{7}{|l|}{ Antecedentes n(\%) } \\
\hline IAM & $66(14.7)$ & $3(25)$ & 0.322 & $61(14)$ & $8(29.6)$ & 0.027 \\
\hline DM & $101(22.4)$ & $4(33.3)$ & 0.374 & $96(22.1)$ & $9(33.3)$ & 0.175 \\
\hline ERC & $39(8.7)$ & $2(16.7)$ & 0.336 & $35(8)$ & $6(22.2)$ & 0.012 \\
\hline HTA & $367(81.6)$ & $10(83.3)$ & 0.875 & $353(81.1)$ & 24(88.9) & 0.314 \\
\hline Falla cardiaca & $131(29.1)$ & $4(33.3)$ & 0.751 & $119(27.4)$ & $16(59.3)$ & $<0.001$ \\
\hline \multicolumn{7}{|l|}{ Síntomas n(\%) } \\
\hline Incremento purulencia esputo & $216(48)$ & $8(66.7)$ & 0.202 & $205(47.1)$ & $19(70.4)$ & 0.019 \\
\hline Incremento producción esputo & $307(68.2)$ & $12(100)$ & 0.019 & $296(68)$ & $23(85.2)$ & 0.062 \\
\hline Incremento de la disnea & 437(97.1) & $12(100)$ & 0.55 & 423(97.2) & $26(96.3)$ & 0.773 \\
\hline Glasgow n(\%) & $1(0.2)$ & $0(0)$ & \multirow{4}{*}{$<0.001$} & $0(0)$ & $1(3.7)$ & \multirow{4}{*}{$<0.001$} \\
\hline 11 & $2(0.4)$ & $0(0)$ & & $2(0.5)$ & $0(0)$ & \\
\hline 14 & $5(1.1)$ & $2(16.7)$ & & $4(0.9)$ & $3(11.1)$ & \\
\hline 15 & $440(98.2)$ & $10(83,3)$ & & 427(98.6) & $23(85.2)$ & \\
\hline \multicolumn{7}{|l|}{ Signos vitales $\mathrm{x}(\mathrm{ds})$} \\
\hline Pulso lat $\mathrm{x} \min$ & $87.7(15.8)$ & $104.5(15.6)$ & $<0.001$ & $87.6(15.6)$ & $96,7(19.64)$ & 0.004 \\
\hline FR resp $x$ min & 20.6(3.9) & $23.2(4.2)$ & 0.026 & $20.6(3.9)$ & $21.6(3.9)$ & 0.195 \\
\hline PAS mm Hg & $136(20.7)$ & $129.5(31.3)$ & 0.285 & $136.3(20.5)$ & $131.3(27.4)$ & 0.237 \\
\hline PAD mm Hg & $77.4(14.4)$ & $77.1(16.4)$ & 0.942 & $77.5(14.4)$ & $75.9(15.4)$ & 0.6 \\
\hline \multicolumn{7}{|l|}{ Paraclínicos x(ds) } \\
\hline $\mathrm{pH}$ & $7.38(0.054)$ & $7.35(0.09)$ & 0.104 & $7.38(0.05)$ & $7.34(0.08)$ & $<0.001$ \\
\hline $\mathrm{PaO}_{2} \mathrm{mmHg}$ & $68.7(18.1)$ & $62.7(24.6)$ & 0.264 & $68.5(17.9)$ & $68.7(23.9)$ & 0.966 \\
\hline $\mathrm{PaCO}_{2} \mathrm{mmHg}$ & $38.1(9.3)$ & $45.8(15.6)$ & 0.006 & $37.9(8.9)$ & $45.7(15.8)$ & $<0.001$ \\
\hline $\mathrm{HCO}_{3}-\mathrm{mmol} / \mathrm{L}$ & $22.2(3.9)$ & $23(5.2)$ & 0.476 & $22.2(3.8)$ & $23.7(5,9)$ & 0.05 \\
\hline $\mathrm{SaO}_{2} \%$ & $90.7(7.1)$ & $81.3(11.8)$ & $<0.001$ & $90.7(7.2)$ & $86.9(10.4)$ & 0.12 \\
\hline $\mathrm{SaO}_{2} \%$ oximetría & $89.9(6.2)$ & $88.3(5.5)$ & 0.383 & $88.8(6.21)$ & $90.5(5.1)$ & 0.589 \\
\hline $\mathrm{PaO}_{2}, \mathrm{FiO}_{2}$ & $244.8(72.9)$ & $178.3(54.4)$ & 0.002 & $245.5(72.6)$ & $204.7(73.2)$ & 0.005 \\
\hline $\mathrm{SaO}_{2} / \mathrm{FiO}_{2}$ gases & $325.5(66.4)$ & $240(76.2)$ & $<0.001$ & $326.7(65.3)$ & $267.2(86.5)$ & $<0.001$ \\
\hline $\mathrm{SaO}_{2} / \mathrm{FiO}_{2}$ oximetría & $322.6(63.9)$ & $260.9(68.5)$ & 0.001 & $323.7(62.8)$ & $277.8(81)$ & $<0.001$ \\
\hline $\mathrm{Hb} \mathrm{mg} / \mathrm{dL}$ & $14(2.1)$ & $13(1.9)$ & 0.078 & $14.1(2)$ & $12.9(2.9)$ & 0.004 \\
\hline Hto $\%$ & $42.5(8.5)$ & $40.6(5.4)$ & 0.324 & $42.6(6.5)$ & $40.4(9,1)$ & 0.104 \\
\hline Creatinina mg/dL & $1(0.84)$ & $1(0.57)$ & 0.88 & $0.99(0.85)$ & $1(0.59)$ & 0.707 \\
\hline BUN mg/dL & $21.4(10.4)$ & $32.9(21.6)$ & $<0.001$ & $21.2(10.3)$ & $29.7(17.2)$ & $<0.001$ \\
\hline Eosinófilos $<50 \mathrm{cel} / \mathrm{mm}^{3} \mathrm{n}(\%)$ & $248(55.1)$ & $9(75)$ & 0.171 & $237(54.7)$ & $20(74.1)$ & 0.047 \\
\hline Consolidación n(\%) & $73(16.2)$ & $3(25)$ & 0.418 & $69(15.9)$ & $7(25.9)$ & 0.171 \\
\hline Fibrilación auricular n(\%) & $33(7.3)$ & $3(25)$ & 0.024 & $30(6.9)$ & $6(22.2)$ & 0.004 \\
\hline
\end{tabular}


Tabla 3. Puntaje de escalas DECAF, BAP65 y CURB65 para ventilación mecánica (VM) y muerte a siete y 30 días.

\begin{tabular}{|c|c|c|c|c|c|c|c|c|c|c|c|}
\hline Escala & & $\begin{array}{c}\text { Población } \\
\text { total } \\
n=462\end{array}$ & $\begin{array}{c}\text { No VM } \\
n=396\end{array}$ & $V M n=66$ & valor $\mathbf{p}$ & $\begin{array}{l}\text { Vivos } \\
n=450\end{array}$ & $\begin{array}{c}\text { Muertos } \\
7 \text { días } \\
n=12\end{array}$ & valor $\mathbf{p}$ & $\begin{array}{l}\text { Vivos } \\
n=435\end{array}$ & $\begin{array}{l}\text { Muertos } \\
30 \text { días } \\
\mathbf{n}=27\end{array}$ & valor $p$ \\
\hline \multirow[t]{6}{*}{ DECAF n(\%) } & 0 & $72(15.6)$ & $71(17.9)$ & $1(1.5)$ & \multirow{6}{*}{$<0.001$} & $71(15.8)$ & $1(8.3)$ & \multirow{6}{*}{0.014} & $71(16.3)$ & $1(3.7)$ & \multirow{6}{*}{$<0.001$} \\
\hline & 1 & $163(35.3)$ & 151(38.1) & $12(18.2)$ & & $162(36)$ & $1(8.3)$ & & $159(36.6)$ & $4(14.8)$ & \\
\hline & 2 & 139(30.1) & $119(30.1)$ & $20(30.3)$ & & 137(30.4) & $2(16.7)$ & & $133(30.6)$ & $6(22.2)$ & \\
\hline & 3 & $59(12.8)$ & $39(9.8)$ & $20(30.3)$ & & $55(12.2)$ & $4(33.3)$ & & $52(12)$ & $7(25.9)$ & \\
\hline & 4 & $25(5.4)$ & $16(4)$ & $9(13.6)$ & & 22(4.9) & $3(25)$ & & 17(3.9) & $8(29.6)$ & \\
\hline & 5 & $4(0.9)$ & $0(0)$ & $4(6.1)$ & & $3(0.7)$ & $1(8.3)$ & & $3(0.7)$ & $1(3.7)$ & \\
\hline \multirow[t]{5}{*}{ BAP65 n $(\%)$} & 1 & $17(3.7)$ & $16(4)$ & $1(1.5)$ & \multirow{5}{*}{0.003} & $17(3.8)$ & $0(0)$ & \multirow{5}{*}{0.027} & $17(3.9)$ & $0(0)$ & \multirow{5}{*}{0.009} \\
\hline & 2 & $289(62.6)$ & $258(65.2)$ & $32(48.5)$ & & $285(63.3)$ & $4(33.3)$ & & $277(63.7)$ & $12(44.4)$ & \\
\hline & 3 & 132(28.6) & 103(26) & $26(39.4)$ & & $128(28.4)$ & $4(33.3)$ & & $124(28.5)$ & $8(29.6)$ & \\
\hline & 4 & $21(4.5)$ & $17(4.3)$ & $6(9.1)$ & & $18(4)$ & $3(25)$ & & 17(3.9) & $4(14.8)$ & \\
\hline & 5 & $3(0.6)$ & $2(0.5)$ & $1(1.5)$ & & $2(0.4)$ & $1(8.3)$ & & $0(0)$ & $3(11.1)$ & \\
\hline \multirow[t]{5}{*}{ CURB65 n(\%) } & 0 & $16(3.5)$ & $15(3,8)$ & $1(1.5)$ & \multirow{5}{*}{0,013} & $16(3.6)$ & $0(0)$ & \multirow{5}{*}{0,058} & $16(3,7)$ & $0(0)$ & \multirow{5}{*}{0.009} \\
\hline & 1 & $228(49.4)$ & $202(51)$ & $26(39.4)$ & & $225(50)$ & $3(25)$ & & $220(50,6)$ & $8(29,6)$ & \\
\hline & 2 & 171(37) & $143(36.1)$ & $26(39.4)$ & & $164(36.4)$ & $7(58.3)$ & & $158(36.3)$ & $13(48,1)$ & \\
\hline & 3 & $43(9.3)$ & $34(8.6)$ & $11(16.7)$ & & $41(9.1)$ & $2(16.7)$ & & $37(8.5)$ & $6(22.2)$ & \\
\hline & 4 & $4(0.9)$ & $2(0.5)$ & $2(3)$ & & $4(0.9)$ & $0(0)$ & & $4(0.9)$ & $0(0)$ & \\
\hline
\end{tabular}

y $\mathrm{SaO}_{2} / \mathrm{FiO}_{2}$ por oximetría mejora considerablemente, los resultados de sensibilidad, especificidad, VPP, VPN, LR+, LR- se muestran en la Tabla 4.

\section{Discusión}

El presente estudio se realizó en pacientes que consultan al servicio de Urgencias del Hospital Militar Central y son diagnosticados con exacerbación de enfermedad pulmonar obstructiva crónica (EPOC). El objetivo fue valorar la relación de saturación arterial de oxígeno en relación con la fracción inspirada de oxígeno $\left(\mathrm{SaO}_{2} / \mathrm{FiO}_{2}\right)$ utilizando la $\mathrm{SaO}_{2}$ obtenida en los gases arteriales y de manera independiente la relación $\mathrm{SaO}_{2} / \mathrm{FiO}_{2}$ con la $\mathrm{SaO}_{2}$ obtenida por oximetría como predictores de mortalidad y requerimiento de ventilación mecánica. Dentro de los resultados encontrados, la población que consulta por exacerbación de la EPOC al Hospital Militar Central de Bogotá, es similar a la reportada en la literatura en cuanto promedio de edad, distribución por sexo, requerimiento de ventilación mecánica y mortalidad (8-10).

La mortalidad de $4.8 \%$ encontrada se encuentra en relación a lo reportado en la literatura y el nivel de complejidad de la institución donde se atienden estos pacientes, como lo mencionan C Roberts y Cols (11) así mismo la necesidad de ventilación mecánica del $14 \%$ se encuentra en el rango a la reportada en pacientes con exacerbación de EPOC y falla ventilatoria $(11,12)$. La mayoría de estudios sobre predictores de desenlace adverso en EPOC tienen en cuenta factores clínicos, en este estudio se encontró que la polipnea y la taquicardia se relacionaron con los desenlaces propuestos, siendo la taquicardia un fuerte predictor clínico de requerimiento de ventilación mecánica y de mortalidad; esto coincide con los reportes de la literatura en que los marcadores clínicos tienen gran relevancia en determinar pronóstico $(13,14)$. También encontramos que es frecuente la presencia de comorbilidades como la falla cardiaca, hipertensión, insuficiencia renal y enfermedad coronaria en pacientes con EPOC, condiciones que también pueden estar relacionadas con los desenlaces del paciente durante su exacerbación.

Los puntajes de severidad de enfermedad más frecuentemente usados en EPOC para predicción de desenlace (DECAF, BAP-65 y CURB-65) se relacionaron con los desenlaces propuestos en el presente estudio, siendo el DECAF el de mejor rendimiento como predictor de desenlaces adversos (15). Este puntaje ha demostrado resultados consistentes para la predicción de ventilación mecánica y mortalidad en diferentes estudios realizados a nivel mundial; sin embargo nuestro objetivo fue evaluar otras herramientas relacionadas con el nivel de oxigenación, que también puede estar alterado en estos pacientes teniendo la consideración geográfica adicional de que los pacientes fueron evaluados a $2600 \mathrm{msnm}(11,16)$. Si bien la oxigenación no es un medidor de uso frecuente a tener en cuenta en los pacientes con la EPOC exacerbada y en la fisiopatología de la enfermedad, 
Tabla 4. Sensibilidad, especificidad, valor predictivo positivo (VPP), valor predictivo negativo (VPN), LR+,LR-, ACOR de SaO , SaO /FiO 2 , puntajes DECAF, BAP-65 y CURB65 para desenlace VM y Mortalidad 30 días.

\begin{tabular}{|c|c|c|c|c|c|c|c|c|}
\hline \multicolumn{9}{|c|}{ Ventilación mecánica } \\
\hline & $\begin{array}{l}\text { Sensibilidad } \\
\text { (IC95\%) }\end{array}$ & $\begin{array}{l}\text { Especificidad } \\
\text { (IC95\%) }\end{array}$ & VPP (IC95\%) & VPN (IC95\%) & $\mathrm{LR}+(\mathrm{IC} 95 \%)$ & LR- (IC95\%) & ACOR (IC95\%) & valor $p$ \\
\hline $\mathrm{SaO}_{2}$ oximetría $<85 \%$ & $15.4(5.84-24.9)$ & $84.6(80.9-88.3)$ & $14.3(5.37-23.2)$ & $85.7(82.1-89.3)$ & $1(0.54-1.85)$ & $1(0.89-1.12)$ & $0.502(0.427-0.577)$ & 0.959 \\
\hline $\mathrm{SaO}_{2} / \mathrm{FiO}_{2}$ oximetría & $84.6(75-94.2)$ & $42(37-47)$ & $19.6(14.8-24.4)$ & $94.3(90.5-98)$ & $1.46(1.28-1.67)$ & $0.37(0.20-1.67)$ & $0.779(0.711-0.847)$ & $<0.001$ \\
\hline $\mathrm{SaO}_{2}$ gases $<85 \%$ & $18.5(8.3-28.7)$ & $83.8(79.9-87.6)$ & $16.2(7.1-25.3)$ & $85.8(82.1-89.5)$ & $1.14(0.65-1.99)$ & $0.97(0.86-1.10)$ & $0.565(0.491-0.639)$ & 0.095 \\
\hline $\mathrm{SaO}_{2} / \mathrm{FiO}_{2}$ gases $<330$ & $83(73.2-92.9)$ & $57(51.9-62.2)$ & $24.8(18.8-30.7)$ & $95.2(92.2-98.2)$ & $1.94(1.65-2.27)$ & $0.3(0.17-0.51)$ & $0.799(0.737-0.861)$ & $<0.001$ \\
\hline DECAF $\geq 2$ & $80.3(69.9-90.7)$ & $56(51-61)$ & $23.4(17.6-29)$ & $94.5(91.3-97.6)$ & $1.83(1.55-2.15)$ & $0.35(0.21-0.58)$ & $0.754(0.692-0.816)$ & $<0.001$ \\
\hline BAP- $65 \geq 3$ & $50(37.2-62.8)$ & $69.2(64.5-73.9)$ & $21.3(14.5-28)$ & $89.3(85.6-92.9)$ & $1.62(1.22-2.15)$ & $0.72(0.56-0.93)$ & $0.605(0.530-0.681)$ & $<0.001$ \\
\hline CURB- $65 \geq 2$ & $59(46.5-71.7)$ & $54.8(49.8-59.8)$ & $17.9(12.6-23.2)$ & $88.9(84.8-93)$ & $1.31(1.04-1.64)$ & $0.75(0.55-1.01)$ & $0.592(0.516-0.668)$ & $<0.001$ \\
\hline \multicolumn{9}{|c|}{ Mortalidad a 30 días } \\
\hline & $\begin{array}{l}\text { Sensibilidad } \\
\text { (IC95\%) }\end{array}$ & $\begin{array}{l}\text { Especificidad } \\
\text { (IC95\%) }\end{array}$ & VPP (IC95\%) & VPN (IC95\%) & LR+ (IC95\%) & LR- (IC95\%) & ACOR (IC95\%) & valor $p$ \\
\hline $\mathrm{SaO}_{2}$ oximetría $<85 \%$ & $11.5(0-25.7)$ & $84.4(80.8-87.9)$ & $4.3(0-9.7)$ & $94(91.5-96.5)$ & $0.74(0.25-2.19)$ & $1.05(0.91-1.21)$ & $0.533(0.414-0.652)$ & 0.573 \\
\hline $\mathrm{SaO}_{2} / \mathrm{FiO}_{2}$ oximetría & $76.9(58.8-95)$ & $39.2(34.4-43.9)$ & 7.1(3.9-10.3) & $96.5(93.5-99.5)$ & $1.26(1.01-1.58)$ & $0.59(0.29-1.20)$ & $0.689(0.568-0,810)$ & 0.001 \\
\hline $\mathrm{SaO}_{2}$ gases $<85 \%$ & $26.9(7.9-45.9)$ & $84(80.5-87.7)$ & $9.5(2.12-16.8)$ & $94.9(92.5-97.3)$ & $1.69(0.87-3.31)$ & $0.87(0.69-1.10)$ & $0.625(0.502-0.747)$ & 0.033 \\
\hline $\mathrm{SaO}_{2} / \mathrm{FiO}_{2}$ gases $<330$ & $80.8(63.7-97.8)$ & $53.2(48.3-58)$ & $9.6(5.5-13.8)$ & $97.8(95.7-99.9)$ & $1.73(1.39-2.14)$ & $0.36(0.16-0.80)$ & $0.732(0.617-0.846)$ & $<0.001$ \\
\hline $\mathrm{DECAF} \geq 2$ & $81.5(64.9-97.9)$ & $52.9(48-57.7)$ & $9.7(5.62-13.8)$ & 97.9(95.8-99.9) & $1.73(1.41-2.12)$ & $0.35(0.16-0.78)$ & $0.763(0.663-0.863)$ & $<0.001$ \\
\hline BAP- $65 \geq 3$ & $55.6(34.9-76.2)$ & $67.8(63.3-72.3)$ & $9.7(4.7-14.7)$ & $96(93.8-98.4)$ & $1.73(1.20-2.48)$ & $0.66(0.43-1.00)$ & $0.658(0.541-0.774)$ & 0.006 \\
\hline CURB- $65 \geq 2$ & $70.4(51.3-89.5)$ & $54.3(49.5-59)$ & $8.7(4.7-12.7)$ & $96.7(94.3-99.2)$ & $1.54(1.18-2.01)$ & $0.55(0.43-1.00)$ & $0.644(0.539-0.748)$ & 0.012 \\
\hline
\end{tabular}

la oxigenación es uno de los últimos factores en afectarse; es conocido que el deterioro de la misma se relaciona con pobre funcionalidad pulmonar y menor sobrevida (17).

El papel de la hipoxemia en las exacerbaciones sigue siendo un papel secundario en relación con otros marcadores de severidad de las exacerbaciones, de hecho no hace parte de ninguno de los puntajes validados para predecir desenlace;sin embargo, es frecuente que los pacientes con exacerbación aguda de EPOC tengan algún grado de hipoxemia y que la suplencia de oxígeno sea frecuente durante las exacerbaciones llegando a ser hasta de $89 \%$ (18). La saturación de oxígeno por gases arteriales se relacionó únicamente con mortalidad a siete días, y coincide con los estudios que indican una relación entre la hipoxemia y mortalidad pero sin significancia estadística. Sin embargo, en este estudio cuando se relaciona la oximetría medida ya sea con oxímetro o por gases arteriales con la $\mathrm{FiO} 2$ administrada al paciente al momento de la toma, sí se encuentra una relación predictora de mortalidad y ventilación mecánica. Hay pocos estudios que hayan evidenciado esta relación, pero algunos si la relacionan con la predicción de una exacerbación y algunos muestran resultados prometedores como monitoreo posterior a una exacerbación y como automonitoreo para decidir el manejo de las exacerbaciones (19).

En este estudio se encuentra que la $\mathrm{SaO}_{2} / \mathrm{FiO}_{2}$ tomada en gases arteriales se relaciona adecuadamente con la tomada con pulso oxímetro coincidiendo con lo encontrado en García-Gutiérrez en 2015 (19, 20); y que los valores bajos se relacionan con los desenlaces negativos de mortalidad y requerimiento de ventilación mecánica. La ventaja de la $\mathrm{SaO}_{2} / \mathrm{FiO}_{2}$ medida por el pulso oxímetro es la ausencia de eventos adversos relacionados con la toma de sangre arterial y la necesidad de dispositivos en ocasiones no disponibles en los niveles de atención básicos. $\mathrm{La} \mathrm{SaO}_{2} / \mathrm{FiO}_{2}$ puede ser una medida de mayor exactitud para valorar la severidad de la hipoxemia, la cual se puede presentar en el paciente con E-EPOC por broncoespasmo severo y otras causas desencadenantes de exacerbación como falla cardiaca, neumonía, neumotórax (21-24).

En cuanto a las limitaciones de este estudio están el no incluir pacientes con exacerbaciones leves que pueden ser evaluados de manera ambulatoria y la realización en 
un centro de urgencias de tercer nivel lo hace sensible al sesgo de espectro que puede aumentar el rendimiento en un estudio de prueba diagnóstica; sin embargo, estos datos permiten proyectar una herramienta que pueda ser validada en el primer nivel de atención para mejorar la calidad del triage y orientar la elección del nivel correcto de atención a estos pacientes (25).

En conclusión nuestro estudio encontró un buen rendimiento para la $\mathrm{SaO}_{2} / \mathrm{FiO}_{2}$ calculados por oximetría y por gases arteriales, para determinar la necesidad de ventilación mecánica y mortalidad en pacientes con exacerbación de la EPOC. No obstante ninguna escala sustituye al juicio médico y la experticia clínica para valorar la situación de cada paciente.

\section{Referencias}

1. Murray CJ, Lopez AD. Alternative projections of mortality and disability by cause 1990-2020: Global Burden of Disease Study. Lancet (London, England). 1997; 349(9064): 1498-504.

2. Lokke A, Lange $\mathbf{P}$, Scharling $\mathbf{H}$, Fabricius $\mathbf{P}$, Vestbo J. Developing COPD: a 25 year follow up study of the general population. Thorax. 2006; 61(11): 935-9.

3. Donaldson GC, Wedzicha JA. COPD exacerbations - 1: Epidemiology. Thorax. 2006; 61(2): 164-8.

4. Echevarria C, Steer J, Heslop-Marshall K, Stenton SC, Hickey PM, Hughes $\mathbf{R}$, et al. Validation of the DECAF score to predict hospital mortality in acute exacerbations of COPD. Thorax. 2016; 71(2): 133-40.

5. Steer J, Gibson J, Bourke SC. The DECAF Score: predicting hospital mortality in exacerbations of chronic obstructive pulmonary disease. Thorax. 2012; 67(11): 970-6.

6. Roche N, Chavaillon J-M, Maurer C, Zureik M, Piquet J. A clinical in-hospital prognostic score for acute exacerbations of COPD. Respiratory Research. 2014; 15(1): 99.

7. Feng Z, Wang T, Liu P, Chen S, Xiao H, Xia N, et al. Efficacy of Various Scoring Systems for Predicting the 28-Day Survival Rate among Patients with Acute Exacerbation of Chronic Obstructive Pulmonary Disease Requiring Emergency Intensive Care. Canadian Respiratory Journal. 2017: 3063510.

8. Shafuddin E, Cooray M, Tuffery C, Hopping S, Sullivan G, Chang C, et al. The CANT score: Predicting mortality in exacerbations of chronic obstructive pulmonary disease. European Respiratory Journal. 2016; 48(suppl 60).

9. Donaldson GC, Mullerova H, Locantore N, Hurst JR, Calverley PM, Vestbo J, et al. Factors associated with change in exacerbation frequency in COPD. Respir Res. 2013; 14:79.

10. Ho TW, Tsai YJ, Ruan SY, Huang CT, Lai F, Yu CJ. In-hospital and one-year mortality and their predictors in patients hospitalized for first-ever chronic obstructive pulmonary disease exacerbations: a nationwide population-based study. PloS one. 2014; 9(12): e114866.

11. Roberts CM, Barnes S, Lowe D, Pearson MG. Evidence for a link between mortality in acute COPD and hospital type and resources. Thorax. 2003; 58(11): 947-9.

12. Esteban A, Anzueto A, Frutos F, Alia I, Brochard L, Stewart TE, et al. Characteristics and outcomes in adult patients receiving mechanical ventilation: a 28-day international study. JAMA. 2002; 287(3): 345-55.

13. Hillas G, Perlikos F, Tzanakis N. Acute exacerbation of COPD: is it the "stroke of the lungs"? 2016; 11: 1579-86.

14. Rusinowicz T, Zielonka TM, Zycinska K. Cardiac Arrhythmias in Patients with Exacerbation of COPD. Advances in experimental medicine and biology. 2017.

15. Shorr AF, Sun X, Johannes RS, Derby KG, Tabak YP. Predicting the need for mechanical ventilation in acute exacerbations of chronic obstructive pulmonary disease: Comparing the CURB-65 and BAP-65 scores. Journal of critical care. 2012; 27(6): 564-70.

16. Singanayagam A, Schembri S, Chalmers JD. Predictors of mortality in hospitalized adults with acute exacerbation of chronic obstructive pulmonary disease. Annals of the American Thoracic Society. 2013; 10(2): 81-9.

17. Qaseem A, Wilt TJ, Weinberger SE, Hanania NA, Criner G, van der Molen T, et al. Diagnosis and management of stable chronic obstructive pulmonary disease: a clinical practice guideline update from the American College of Physicians, American College of Chest Physicians, American Thoracic Society, and European Respiratory Society. Annals of internal medicine. 2011; 155(3): 179-91.

18. Cameron L, Pilcher J, Weatherall M, Beasley R, Perrin K. The risk of serious adverse outcomes associated with hypoxaemia and hyperoxaemia in acute exacerbations of COPD. Postgraduate medical journal. 2012; 88(1046): 684-9.

19. Garcia-Gutierrez S, Unzurrunzaga A, Arostegui I, Quintana JM, Pulido E, Gallardo MS, et al. The Use of Pulse Oximetry to Determine Hypoxemia in Acute Exacerbations of COPD. COPD. 2015; 12(6): 613-20.

20. Kelly AM, McAlpine R, Kyle E. How accurate are pulse oximeters in patients with acute exacerbations of chronic obstructive airways disease? Respiratory medicine. 2001; 95(5): 336-40.

21. Runciman WB, Webb RK, Barker L, Currie M. The Australian Incident Monitoring Study. The pulse oximeter: applications and limitations--an analysis of 2000 incident reports. Anaesthesia and intensive care. 1993; 21(5): 543-50.

22. Laishley RS, Aps C. Tension pneumothorax and pulse oximetry. British journal of anaesthesia. 1991; 66(2): 250-2.

23. Masip J, Gaya M, Paez J, Betbese A, Vecilla F, Manresa R, et al. Pulse oximetry in the diagnosis of acute heart failure. Revista Española de Cardiología (English ed). 2012; 65(10): 879-84

24. Martínez GM, Casas DP, Bastidas AR, Oliveros H, Pinilla PA, Calderón WJ, et al. Índices de oxigenación como predictores de ventilación mecánica en neumonía a 2600 metros de altitud. Acta Médica Colombiana. 2016; 41(3).

25. Dalbak LG, Straand J, Melbye H. Should pulse oximetry be included in GPs' assessment of patients with obstructive lung disease? Scandinavian journal of primary health care. $2015 ; \mathbf{3 3 ( 4 )}$ : 305-10. 